\title{
GENOME-WIDE CHARACTERIZATION AND ANALYSIS OF SBP TRANSCRIPTION FACTOR FAMILY IN COMMON BEAN (PHASEOLUS VULGARIS L.)
}

\author{
ILHAN, E. \\ Department of Molecular Biology and Genetics, Erzurum Technical University \\ Erzurum, Turkey \\ (e-mail: emre.ilhan@erzurum.edu.tr; phone:+90-444-5388-2387)
}

(Received $6^{\text {th }}$ Jul 2018; accepted $14^{\text {th }}$ Aug 2018)

\begin{abstract}
SQUAMOSA promoter binding proteins (SBPs) are considered as being a major family of plant-specific transcription factors which plays an important role in flower improvement process. To discover SBP genes in common bean [Phaseolus vulgaris (L.)] genome, in silico analysis methods were carried out in this study. Twenty-three members of Pvul-SBP gene family were identified from common bean genome. A phylogenetic tree drawn using SPB proteins of Phaseolus vulgaris, Arabidopsis thaliana and Glycine max was separated into three main groups (A, B, and C). The C group was clustered into nine subclades. The chromosome locations of Pvul-SBP genes showed that Pvul-SBP-1/Pvul-SBP-6, PvulSBP-2/Pvul-SBP-16, Pvul-SBP-5/Pvul-SBP-14 and Pvul-SBP-9/Pvul-SBP-15 gene couples were segmentally-duplicated. Also, orthologue genes were defined using SBP proteins of Arabidopsis thaliana and Glycine max. The expression levels of Pvul-SBP genes in different plant tissues such as roots, leaf and floral organs showed some of $P v u l-S B P$ genes were up or down regulated in certain tissue types. The results of this research would provide the estimation of the evolutionary relationships between $S B P$ genes in various plants such as P. vulgaris, G. max and A. thaliana.
\end{abstract}

Keywords: gene expression, orthologue relationship, phylogeny, synteny

\section{Introduction}

Identification and characterization of transcription factor (TF) families in plants has been turned into one of the most important topics for plant scientists in recent years due to their importance in regulation networks of plant growing processes (Buyuk et al., 2016; Inal et al., 2017; Ilhan et al., 2018). There have been many well-conducted studies for characterization of several plant-specific TFs in many plant species. However, there is no information about some of them including SBP (SQUAMOSA promoter binding protein family) in certain plant species (Wang et al., 2015).

SBPs are considered as being a major family of plant-specific transcription factors which plays an important role in flower improvement processes (Pan et al., 2017). SBP domain, a highly-conserved DNA-binding domain, has specific properties for each member of this TF family. Even though this domain is known to be a zinc-binding domain, structures within this domain are not similar to those ones in other zinc-binding domains and thus has been declared as representing a novel type of zinc binding motif (Wang et al., 2015).

Recent studies which were aimed at defining the roles of SBP family of transcription factors showed divergent roles of them in plant developmental processes (Zhang et al., 2016).

Among sixteen identified SBP genes in Arabidopsis genome, many of them have been revealed to play role in embryogenesis (Unte et al., 2003), development of shoots and leaves (Schwarz et al., 2008), flowering process (Gandikota et al., 2007), transition 
between vegetative and generative phases (Jung et al., 2012) and plant hormone signaling (Zhang et al., 2007)

Genome-wide characterization and expression studies of SBP genes have been conducted in a number of plant species including Betula pendula, Chlamydomonas, Oryza sativa, Zea mays, Populus trichocarpa, L. esculentum, Malus $\times$ domestica Borkh., Salvia miltiorrhiza, Gossypium hirsutum, Arachis hypogaea L., petunia, Capsicum annuum L., Chrysanthemum morifolium, Phyllostachys edulis, Solanum tuberosum, Ziziphus jujuba and (Lannenpaa et al., 2004; Kropat et al., 2005; Xie et al., 2006; Chuck et al., 2010; Lu et al., 2011; Salinas et al., 2012; Li et al., 2013; Zhang et al., 2014, 2015, 2016; Li et al., 2016; Preston et al., 2016; Song et al., 2016; Pan et al., 2017; Kavas et al., 2017; Shao et al., 2017).

The common bean [Phaseolus vulgaris (L.)] is a self-pollinated legume species. It has a small genome size of $588 \mathrm{Mbp}$ and a diploid genome $(2 \mathrm{n}=2 \mathrm{x}=22)$ (Arumuganthan and Earle, 1991). It is one of the earliest plant species cultivated. As a result of its nitrogen fixation ability, it enriches the nitrogen content of soil. It has an important role in human nutrition, especially in developing countries, due to its high protein content (Broughton et al., 2003). It has been possible to analyze and characterize the SBP gene family with the publication of the whole genome sequence of common bean by Schmutz in 2014 (Schmutz et al., 2014). However, the SPB proteins in Phaseolus vulgaris L. have not been identified and characterized yet. To fill this gap in the literature and due to importance of this gene family in plant developmental processes, this study aimed at describing and characterizing SBP proteins in Phaseolus vulgaris L. Accordingly, 23 Phvul-SBP members were identified and comprehensive analysis of the sequence phylogeny, genomic organization, exon-intron architecture variation, conserved protein motifs, gene duplication events, and expression analysis were performed.

\section{Materials and methods}

\section{Identification of SBP genes in P. vulgaris genome}

Phaseolus vulgaris of SBP protein sequences were retrieved from Phytozome database v12.1 (www.phytozome.net) using keyword searching with Pfam Accession Number (PF03110) obtained from Pfam Database (http://pfam.xfam.org). BLASTP and BLASTX searches (National Center for Biotechnology Information [NCBI]: http://www.ncbi.nlm.nih.gov) was used to confirm Pvul-SBP proteins. Non-redundant sequences were obtained using decrease redundancy tool (http://web.expasy.org/decrease_redundancy/). SBP domains in non-redundant sequences were checked by HMMER (http://www.ebi.ac.uk). The solid and chemical traits of SBP proteins in Phaseolus vulgaris, such as the theoretical isoelectric point (pI), the number of amino acids, and the molecular weight (Da), were identified using the ProtParam tool (http://web.expasy.org/protparam/).

\section{Phylogenetic analysis, physical location, conserved motifs of phvul-SBP genes, gene structure and gene duplication events}

Chromosomal locations and CDS sizes (bp) were identified by using Phytozome database v12.1. The Pvul-SBP genes were mapped with MapChart (Voorrips, 2002). 
Multiple sequence alignment of Pvul-SBP proteins was conducted with ClustalW. Phylogenetic analysis was performed using MEGA v7 (Tamura et al., 2013) and Neighbor-joining (NJ) algorithm with 1000 replicated-bootstrap value.

The conserved motifs in Pvul-SBP protein sequences were identified using the MEME (Multiple Expectation Maximization for Motif Elucidation) online tool (Bailey et al., 2006). The limits for maximum number of motifs and minimum/maximum width were adjusted as 20 and 2, 50, respectively. Motif sites were among 2 and 300. Site distribution was set as any number of repetitions. The described conserved motifs were examined in InterProscan with default adjusting (Quevillon et al., 2005).

Online Gene Structure Display Server program tool (GSDS) was used (Guo et al., $2007)$ to predict the exon/intron organization of the Pvul-SBP genes. Genomic DNA sequences and coding sequences of Pvul-SBP genes were utilized.

Segmental duplicate gene pairs were examined on the Plant Genome Duplication Database server (PGDD) (Lee et al., 2013), with a display range of $100 \mathrm{~kb}$. The nonsynonymous rates $(\mathrm{Ka})$, synonymous rates $(\mathrm{Ks})$ and developmental constraints $(\mathrm{Ka} / \mathrm{Ks})$ with the duplicated pairs of Pvul-SPLs were evaluated using the CODEML program in PAML (Yang, 2007). The roughly date of the duplication events was determined using $\mathrm{T}=\mathrm{Ks} / 2 \lambda \times 10^{-6}$ Mya (million years ago), in terms of the synonymous substitution rates $(\lambda)$ in common bean of $8.46 \times 10^{-9}$ (Schmutz et al., 2014).

\section{Synteny analysis}

P. vulgaris and A. thaliana, P. vulgaris and G. max of orthologue SBP genes were identified with PGDD (Lee et al., 2013). Then, the protein sequences of orthologue genes were retrieved from Phytozome v12.1. The retreived synteny map was created using iTAK - Plant Transcription factor \& Protein Kinase Identifier and Classifier (Zheng et al., 2016).

\section{Gene expression analysis in silico}

The expression levels of Pvul-SBP genes examined in special tissue libraries of plants at different stages of growth, including root_10 (10 days after planting), nodules, root_19 (19 days after planting), young pods, stem_10 (10 days after planting), stem_19 (19 days after planting), green mature buds, leaves, young triloliates, flower buds and flowers were retrieved from Phytozome Database v12.1 (http://phytozome.jgi.doe.gov/pz/portal.html\#!info?alias=Org_Pvulgaris). $\quad$ FPKM (expected number of fragments per kilobase of transcript sequence per millions base pairs sequenced) units were used for the expression levels in silico. FPKM values were $\log 2$ transformed and the heatmap was produced with the algorithm CIMMiner (http://discover.nci.nih.gov/cimminer).

\section{Results and discussion}

\section{Identification of SBP gene family in common bean}

Sequences of SBP proteins in the $P$. vulgaris genome were downloaded from Phytozome database v12.1 (www.phytozome.net) using keyword searching with Pfam Accession Number (PF03110) retrieved from Pfam Database. Subsequently, SBP domains were analyzed by searching HMMER and Pfam databases in candidate Phvul- 
SBP proteins and the redundant sequences were discarded after the confirmation. A total of 23 candidate Phvul-SBP genes in Phaseolus vulgaris genome was discovered and some descriptive information was given in Table 1 which involves the information about chromosomal location, the coding sequences (CDS, lengths), amino acid (length), molecular weight and number of isoelectric point (pI). As shown in Table 1 and Figure 1, all of the non-redundant Phvul-SBP genes were distributed on 10 chromosomes of P. vulgaris. While the lowest number of Phvul-SBP genes was obtained on chromosome 11 (one Phvul-SBP gene), the highest number of Phvul-SBPs was found on chromosome 3 (4 Phvul-SBP genes) (Fig. 1). The CDS of the Phvul-SBP genes extended from 378 (Phvul-SBP-4) to 3210 (Phvul-SBP-9). The length of Phvul-SBP proteins extended from 125 (Phvul-SBP-4) to 1039 (Phvul-SBP-9) amino acids (aa). pIs of Phvul-SBP proteins were among 5.8 (Phvul-SBP-15) and 9.46 (Phvul-SBP-12) ranging from acidic to alkaline, and the molecular weight of Phvul-SBPs were between 14756.09 Da (Phvul-SBP-14) and 114838.01 Da (Phvul-SBP-9) (Table 1). SBP gene family which is essential to plants was detected and classified in various species such as Arabidopsis (Rhoades et al., 2002), rice (Xie et al., 2006), tomato (Salinas et al., 2012), Citrus (Shalom et al., 2015), Gossypium raimondii (Ali et al., 2017), wheat (Wang et al., 2015), maize (Mao et al., 2016) and grape (Hou et al., 2013).

Table 1. The information of 23 Phvul-SBP proteins

\begin{tabular}{|c|c|c|c|c|c|c|}
\hline Gene ID & Phytozome ID & Chromosomal location & CDS length & aa length & Molecular weight (kDa) & pI \\
\hline Phvul-SBP-1 & Phvul.001G091400 & Chr01:15552500..15556152 & 1095 & 364 & 40.66 & 7.16 \\
\hline Phvul-SBP-2 & Phvul.001G141000 & Chr01:38299114..38303396 & 1074 & 357 & 38.20 & 8.97 \\
\hline Phvul-SBP-3 & Phvul.001G262600 & Chr01:50971750..50975439 & 1362 & 453 & 49.63 & 7.58 \\
\hline Phvul-SBP-4 & Phvul.002G114500 & Chr02:24504136..24505511 & 378 & 125 & 13.99 & 9.19 \\
\hline Phvul-SBP-5 & Phvul.002G230300 & Chr02:40279842..40282041 & 510 & 169 & 19.49 & 5.47 \\
\hline Phvul-SBP-6 & Phvul.002G286000 & Chr02:45529658..45533339 & 1152 & 383 & 41.91 & 8.82 \\
\hline Phvul-SBP-7 & Phvul.003G008800 & Chr03:989197..995182 & 3030 & 1009 & 111.88 & 8.45 \\
\hline Phvul-SBP-8 & Phvul.003G039600 & Chr03:4337189..4341787 & 1443 & 480 & 53.96 & 6.4 \\
\hline Phvul-SBP-9 & Phvul.003G120700 & Chr03:30548630..30555522 & 3120 & 1039 & 114.84 & 7.35 \\
\hline Phvul-SBP-10 & Phvul.003G182900 & Chr03:40612076..40617217 & 1650 & 549 & 60.30 & 6.49 \\
\hline Phvul-SBP-11 & Phvul.005G074000 & Chr05:13162582..13164100 & 576 & 191 & 21.61 & 8.86 \\
\hline Phvul-SBP-12 & Phvul.005G101900 & Chr05:31789454..31791276 & 510 & 169 & 19.30 & 9.46 \\
\hline Phvul-SBP-13 & Phvul.006G159700 & Chr06:26373187..26387876 & 2289 & 762 & 85.64 & 6.85 \\
\hline Phvul-SBP-14 & Phvul.006G183900 & Chr06:28501741..28503445 & 384 & 127 & 14.76 & 6.45 \\
\hline Phvul-SBP-15 & Phvul.007G210600 & Chr07:33265157..33272512 & 3045 & 1014 & 112.51 & 5.8 \\
\hline Phvul-SBP-16 & Phvul.007G258400 & Chr07:37986117..37990987 & 1107 & 368 & 39.58 & 8.76 \\
\hline Phvul-SBP-17 & Phvul.008G157100 & Chr08:28607666..28613632 & 1035 & 344 & 37.55 & 9.15 \\
\hline Phvul-SBP-18 & Phvul.008G157200 & Chr08:28427029..28429343 & 951 & 316 & 34.55 & 8.82 \\
\hline Phvul-SBP-19 & Phvul.009G165100 & Chr09:24615068..24618431 & 1194 & 397 & 43.71 & 7.67 \\
\hline Phvul-SBP-20 & Phvul.009G219200 & Chr09:33103356...33114413 & 3096 & 1031 & 113.97 & 6.59 \\
\hline Phvul-SBP-21 & Phvul.010G056000 & Chr10:10002440..10005618 & 993 & 330 & 36.35 & 8.85 \\
\hline Phvul-SBP-22 & Phvul.010G056200 & Chr10:10088279..10091188 & 1119 & 372 & 41.02 & 6.9 \\
\hline Phvul-SBP-23 & Phvul.011G164800 & Chr11:46749842..46751202 & 561 & 186 & 20.80 & 8.97 \\
\hline
\end{tabular}

pI: Theoretical isoelectric point, kDa: kiloDalton 


\section{$\begin{array}{llllll}\text { Pvul_Chr01 } & \text { Pvul_Chr02 } & \text { Pvul_Chr03 } & \text { Pvul_Chr05 } & \text { Pvul_Chr06 } & \text { Pvul Chr07 }\end{array}$}
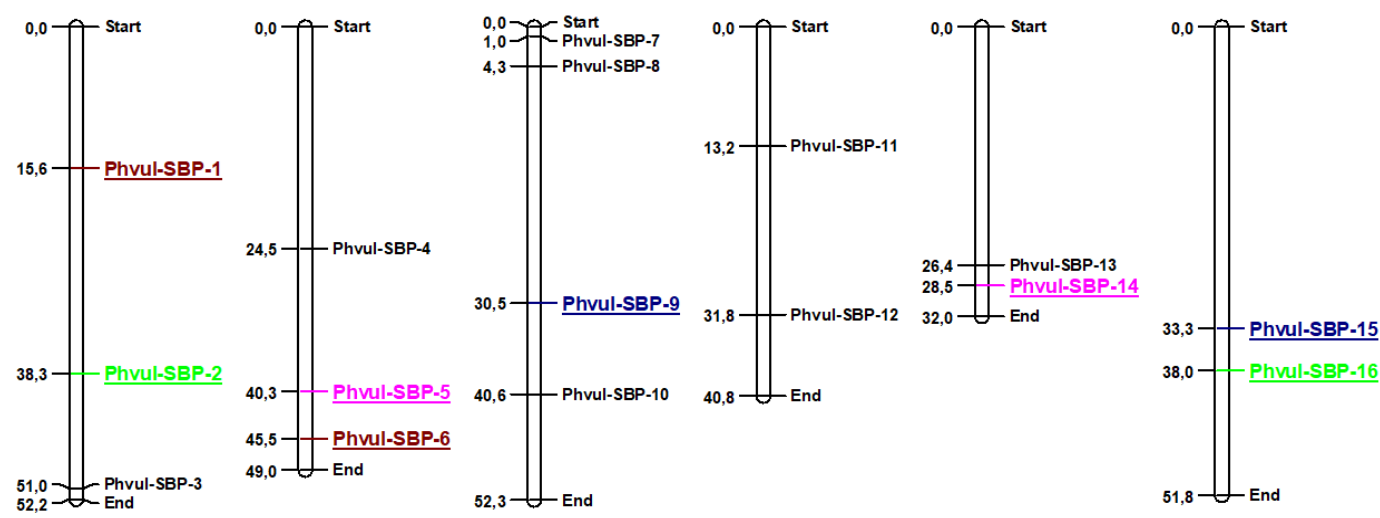

Pvul_Chr08

Pvul_Chr09

Pvul_Chr10

Pvul_Chr11
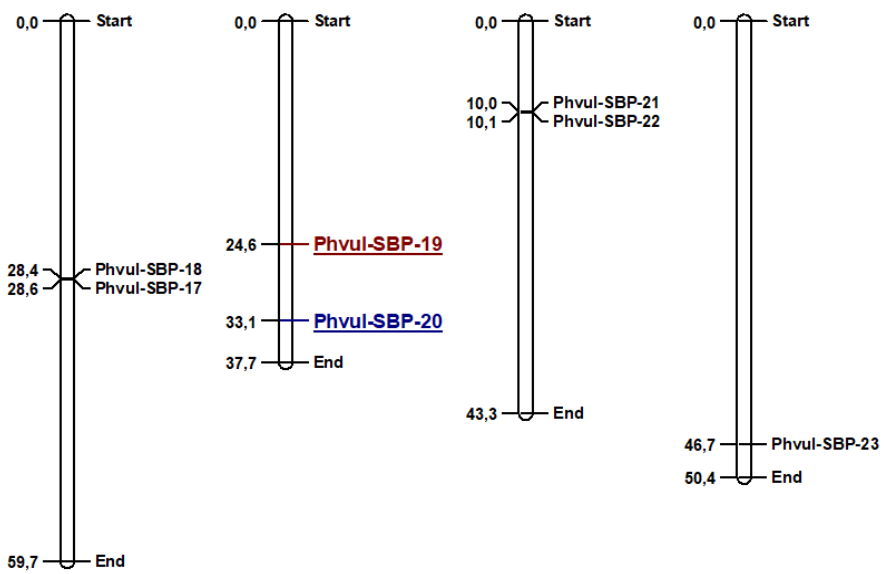

Figure 1. Distribution of Phvul-SBP genes on common bean chromosomes. Similar colors present segmentally - duplicated genes

Several paralogous gene pairs are originated from gene duplication events (Cannon et al., 2004). Furthermore, organisms adapt to different environments via gene duplication events throughout processes of development and growth (Bowers et al., 2003; Gu et al., 2003). Plant genomic structure, which can occur by independent events involving in segmental or tandem duplications, is one of most important characteristics of gene duplication (Flagel and Wendel, 2009). Based on significance of gene duplications on evolution of gene families in plants, the gene duplication events of putative Phvul-SBP genes in common bean genome have been examined. After the duplication analysis, six segmentally-duplicated gene couples (Phvul-SBP-1/PhvulSBP-19, Phvul-SBP-2/Phvul-SBP-16, Phvul-SBP-5/Phvul-SBP-14, Phvul-SBP-6/PhvulSBP-19, Phvul-SBP-9/Phvul-SBP-15 and Phvul-SBP-9/Phvul-SBP-20) among the identified 23 Phvul-SBP genes in common bean (Table 2) were detected. According to the gene duplication analysis, none of the gene couples have been found to include tandem repeats. 
The $\mathrm{Ka} / \mathrm{Ks}$ ratio has been become a popular parameter for genome-wide identification researches of gene families in recent years. This ratio gives a worthwhile information about the selective evolutionary pressures that are acting on that gene (Yang and Swanson, 2002; Kryazhimskiy and Plotkin, 2008). According to Juretic et al. (2005), a Ka/Ks larger than 1 reflects the positive selection during the evolution of the gene sequence. Additionally, a $\mathrm{Ka} / \mathrm{Ks}$ lower than 1 indicates duplications have undergone purifying selection and a $\mathrm{Ka} / \mathrm{Ks}=1$ means neutral selection in duplication events. As displayed in Table 2, the divergence time of segmentally duplicated PhvulSBP gene pairs extended from 32.77 to 101.61 million years (MYA). Except for one, all of the duplicated Phvul-SBP gene pairs showed Ka/Ks ratio lower than 1 which reflects the occurrence of purifying selection after the duplication events. The Ka/Ks ratio $(=1)$ of Phvul-SBP-1/Phvul-SBP-19 indicated the predominance of neutral selection during duplication events of this gene pair (Table 2).

Table 2. The Ka/Ks ratios and identified divergence times for the segmentally duplicated Phvul-SBP proteins

\begin{tabular}{c|c|c|c|c|c}
\hline \multicolumn{2}{c|}{ Paralogous gene pairs } & Ka & Ks & Ka/Ks & Duplication date (MYA) \\
\hline Phvul-SBP-1 & Phvul-SBP-19 & 0 & 0 & 1 & - \\
\hline Phvul-SBP-2 & Phvul-SBP-16 & 0.1922 & 0.8346 & 0.2075 & 49.33 \\
\hline Phvul-SBP-5 & Phvul-SBP-14 & 0.3358 & 1.0685 & 0.0917 & 63.15 \\
\hline Phvul-SBP-6 & Phvul-SBP-19 & 0.2174 & 0.7511 & 0.2834 & 44.39 \\
\hline \multirow{2}{*}{ Phvul-SBP-9 } & Phvul-SBP-15 & 0.3989 & 1.7193 & 0.1484 & 101.61 \\
\cline { 2 - 6 } & Phvul-SBP-20 & 0.1836 & 0.5544 & 0.2868 & 32.77 \\
\hline
\end{tabular}

\section{Phylogenetic analysis, gene structure and conserved motifs of Phvul-SBPs}

To discover the relationships between Phvul-SBP proteins, a phylogenetic tree of SBP domain proteins in Phaseolus vulgaris, Arabidopsis and Glycine max was structured using amino acid sequences with contributed bootstrap values (1000 replicates; Fig. 2) via neighbour joining method. Phvul-SBP proteins were clustered into twelve groups, A - C9 (Fig. 2). Similar coding and exon-intron sequences were observed in most of the Phvul-SBP genes which were found in the same subgroup in phylogenetic tree.

To investigate conserved motifs in Phvul-SBP proteins, MEME (v5.0.1) was used. MEME software finds the most statistically significant (low E-value) motifs based on its log likelihood ratio, its width and number of occurrences, the background letter frequencies and the size of the training set. Motifs with E-values larger than $0.01(1 \mathrm{e}-2)$ are possibly just statistical artifacts, and not real motifs (Bailey et al., 2009).

A total of 20 conserved motifs were described (Fig. 3 and Table 3). The lengths of identified motifs were between 8 and 50 amino acids. A total of Phvul-SBP proteins consist of Motif 1 which is one of the two motifs which have SBP domain among all motifs. A similar result was found in Ali et al. (2017).

Twenty-three common bean SBP gene structures were investigated and the estimated number of exons among the 23 Phvul-SBP genes extended from two to 10 (Fig. 4). As seen in Figure 4, these genes were also clustered into twelve groups in terms of the gene structure analysis in line with the phylogenetic tree of Phvul-SBP proteins shown in Figure 2. Deep examinations of the segmentally duplicated gene pairs, which have 
same exon-intron structures and similar motifs, would contribute to a comprehension of the different roles they play in development and growth. In a recent research, it has been reported that the gene pairs, which have similar motif sequences and orders could show similar functions in moso bamboo (Pan et al., 2017). These gene pairs could be originated from an ancestor in evolutionary process.

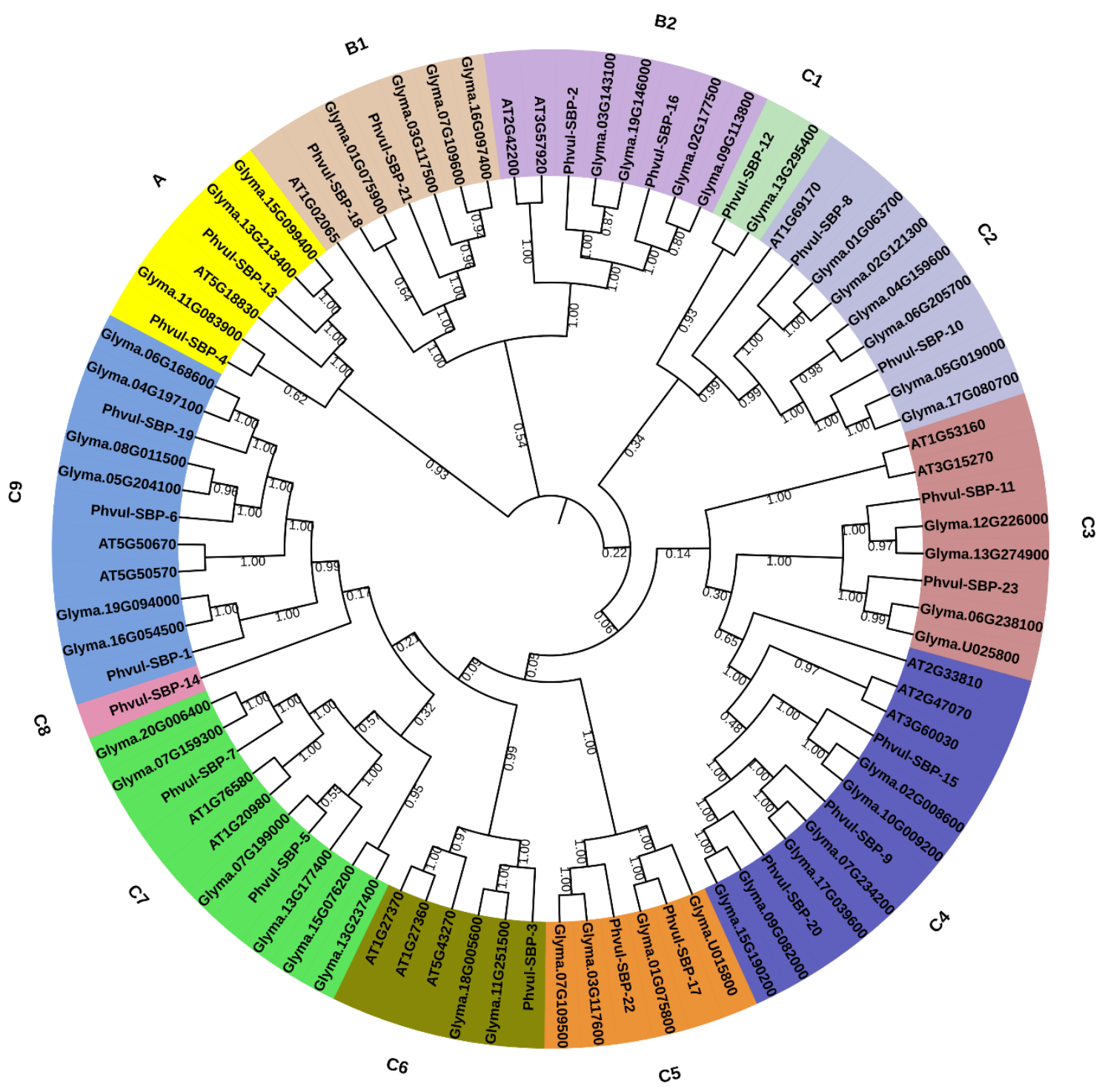

Figure 2. A phylogenetic tree of SBP proteins of common bean, soybean and Arabidopsis thaliana. The tree was generated with MEGA $v 7$ and Neighbor-joining (NJ) algorithm with 1000 replicated-bootstrap values

Table 3. The MEME motif sequences and length of Pvul-SBP proteins

\begin{tabular}{c|c|c|c|c|c}
\hline Motif ID & Conserved sequence & E-value $^{*}$ & Sites & Width & Domain \\
\hline 1 & $\begin{array}{c}\text { RCQVEGCNADLSNAKDYHRRHKVCEMHSKAP } \\
\text { KVJVAGLEQRFCQQCSRFH }\end{array}$ & $2.3 \mathrm{E}-730$ & 23 & 50 & SBP \\
\hline 2 & VLSEFDEGKRSCRRRLAGHNERRRKPQPE & $5.5 \mathrm{E}-351$ & 22 & 29 & SBP \\
\hline 3 & $\begin{array}{c}\text { RTDRIVFKLFGKDPNDFPLVLRAQILNWLSHSP } \\
\text { TEIESYIRPGCIILTIY }\end{array}$ & $5 \mathrm{E}-92$ & 5 & 50 & Unknown \\
\hline
\end{tabular}




\begin{tabular}{c|c|c|c|c|c}
\hline 4 & $\begin{array}{c}\text { PAGLTPLHVAASISGSDNVLDALTDDPGMVGIE } \\
\text { AWKSARDSTGLTPHDYA }\end{array}$ & $8 \mathrm{E}-48$ & 4 & 50 & Unknown \\
\hline 5 & $\begin{array}{c}\text { TAMVYRPAMLSMVAIAAVCVCVALLFKSSPKV } \\
\text { YYVFRPF }\end{array}$ & $7 \mathrm{E}-41$ & 4 & 39 & Unknown \\
\hline 6 & $\begin{array}{c}\text { FIQEMGWLLHRSRLKVRLGPVAPIQDJFQFNRF } \\
\text { KWLVDFSMDHDWCAVMK }\end{array}$ & $3 \mathrm{E}-34$ & 3 & 50 & Unknown \\
\hline 7 & GKRSLEWDLNDWKWDGDLFTASRLNSVPSDC & $1 \mathrm{E}-24$ & 3 & 31 & Unknown \\
\hline 8 & $\begin{array}{c}\text { PKILCVKPLAVPASKRAQFIVKGVNLLQPATRL } \\
\text { LCALEGKYLVCE }\end{array}$ & $1 \mathrm{E}-24$ & 5 & 45 & Unknown \\
\hline 9 & FWRTGWVYVRVQHQLAFLYNGQVVIDVPL & $1 \mathrm{E}-21$ & 4 & 29 & unknown \\
\hline 10 & DSSCALSLLSSQSW & $2 \mathrm{E}-16$ & 10 & 14 & Unknown \\
\hline 11 & SLIGLKLGKRIYFED & $5 \mathrm{E}-15$ & 9 & 15 & Unknown \\
\hline 12 & ALLEMGLLHKAVKRNSRPMVELLLRYVP & $1 \mathrm{E}-13$ & 4 & 28 & Unknown \\
\hline 13 & PVIVAEEEICSEICTLENVJE & $2 \mathrm{E}-11$ & 5 & 21 & Unknown \\
\hline 14 & $\begin{array}{c}\text { YLLMSLLRILSNMHSNGSDHTTBQDILSHLLRN } \\
\text { LASLAGPNNG }\end{array}$ & $2 \mathrm{E}-13$ & 3 & 43 & Unknown \\
\hline 15 & HJKFSCTIPNVVGRGFIEVED & $2 \mathrm{E}-10$ & 5 & 21 & Unknown \\
\hline 16 & WEELDYGT & $6 \mathrm{E}-09$ & 10 & 8 & n/a \\
\hline 17 & LRGHYSYIQLVQKKINKKGGAAHVVDIP & $1 \mathrm{E}-08$ & 3 & 28 & Unknown \\
\hline 18 & WVQCDSLKSSPPQTSRNSDSTSTQ & $9 \mathrm{E}-09$ & 3 & 24 & Unknown \\
\hline 19 & $\begin{array}{c}\text { MDWNLKAPSWDLVEVDKANJPNIESMEEHNRF } \\
\text { GMFRMEGEFSVDLKLGQV }\end{array}$ & $2 \mathrm{E}-07$ & 2 & 50 & Unknown \\
\hline 20 & CRQADERGRIQMNJPRNSGYKSFHIR & $5 \mathrm{E}-06$ & 3 & 26 & Unknown \\
\hline
\end{tabular}

*The expected value of each motif prediction was given in the MEME program
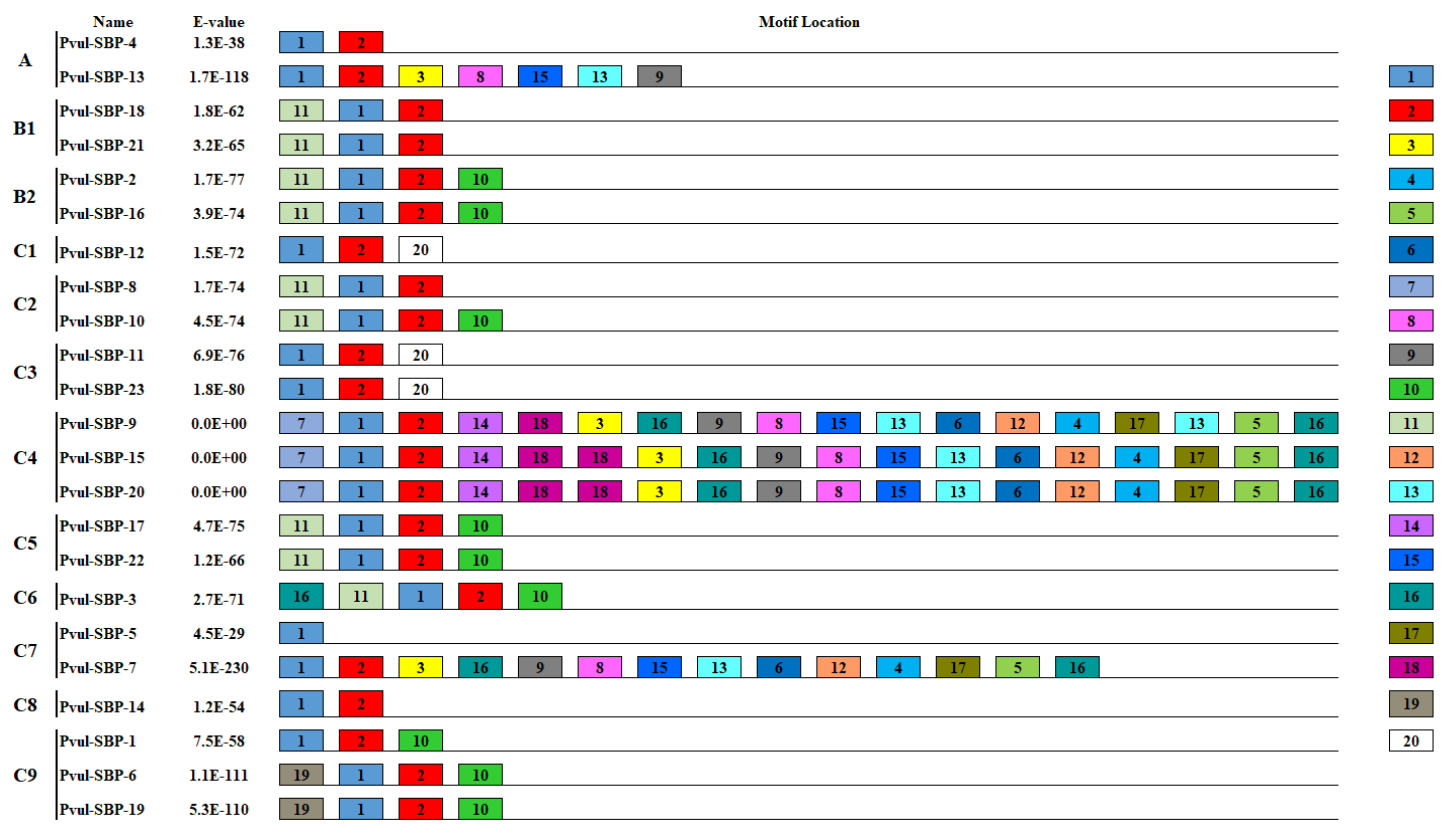

Figure 3. Shematic representation of the 20 conserved motifs in Pvul-SBP proteins. The 20 conserved motifs were classified using MEME Suite v5.0.1. Each motifs were shown by different colored boxes. The numbers in the boxes represent motif 1 -motif 20, respectively 

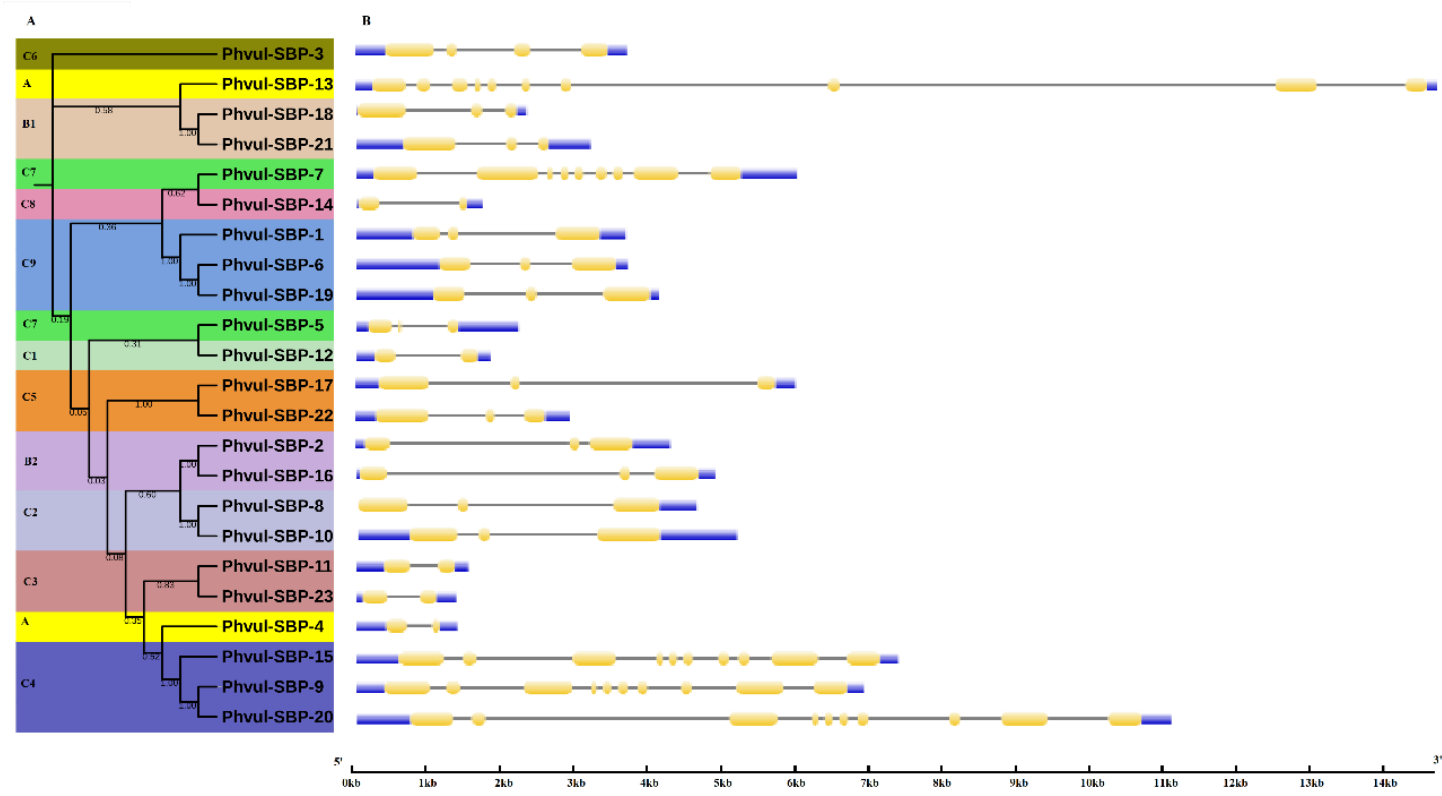

Figure 4. Gene structure of Phvul-SBP genes. A. The number of below branches of the phylogenetic tree display bootstrap values. The tree was structured with MEGA $v 7$ by the Neighbor-Joining (NJ) method with 1000 bootstrap replicates. The A-C9 numerals on the tree correspond to 12 Pvul-SBP groups shown in Figure 2. B. Yellow colors represent exons, grey lines represent introns and blue colors represent untranslated regions (UTRs)

\section{Comparative and synteny events among SBP genes of $P$. vulgaris, Arabidopsis thaliana and Glycine max}

To calculate evolutionary relationship of the $S B P$ gene family in $P$. vulgaris, Arabidopsis and Glycine max, the synteny events among SBP protein sequences of these plants are shown in Figure 5.
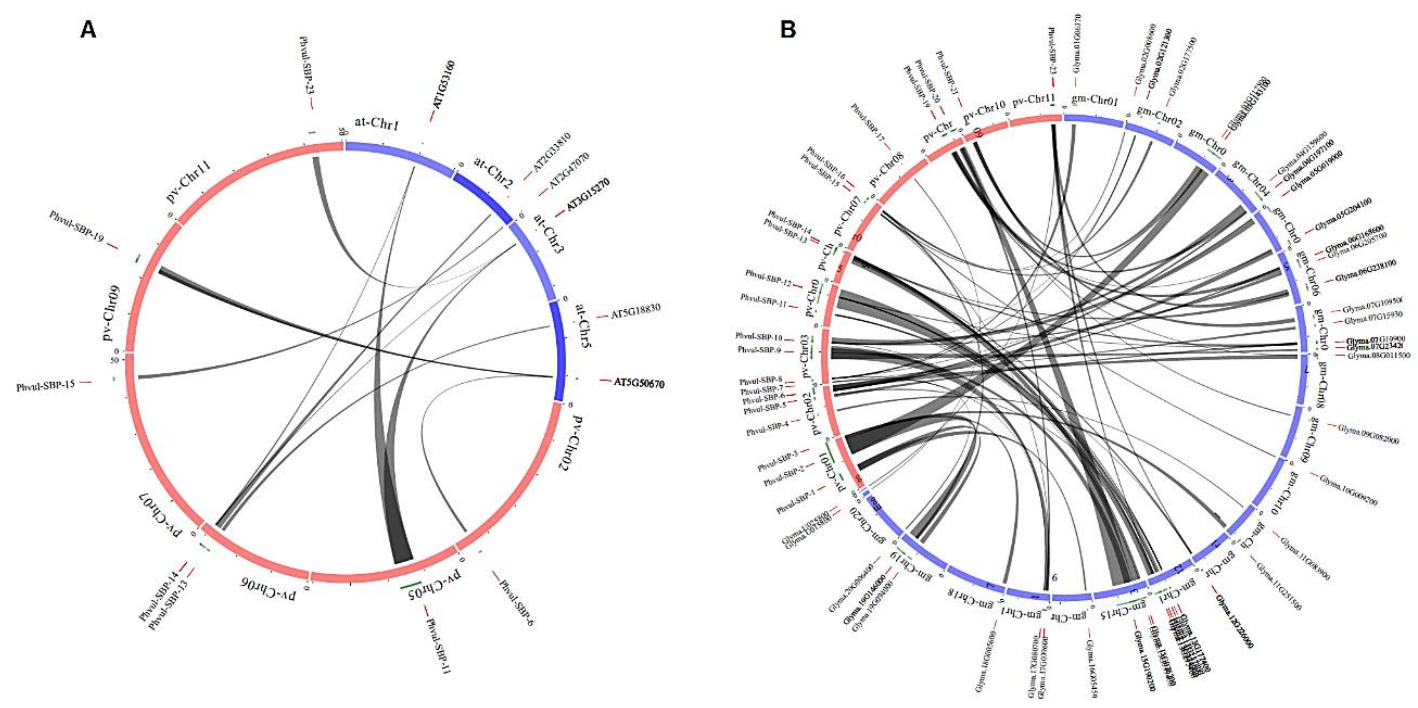

Figure 5. Genome wide synteny analysis of SBP genes. A. Comparative map between $P$. vulgaris and A. thaliana. B. Comparative map between P. vulgaris and G. max 
This synteny analysis was established on SBP proteins of 6 Arabidopsis and 40 Glycine max. Since P. vulgaris and G. max were closely related genomes (Schmutz et al., 2014), $S P B$ genes of these plants showed high similarity. However, 22 Pvul-SBP genes were matched to $40 G$. max $S B P$ genes distributed on all chromosomes of $G$. max. In a recent study, it was reported that some Arabidopsis SBP genes were syntenic to those of diploid cotton genes and these genes showed an evolutionary relationship (Ali et al., 2017). In another study, the synteny analysis between apple and Arabidopsis demonstrated that 11 apple and Arabidopsis genes were located in the duplicated genomic regions. In the same study, it was notified that duplications such as segmental, tandem and genomic duplications have important roles in evolutionary processes ( $\mathrm{Li}$ et al., 2013).

\section{Expression profiles of Phvul-SBPs in different tissues}

In this research, a common mRNA analysis of Phvul-SBP genes were performed via publicly available expression data in Phytozome v12.1 online plant genomics resource (https://phytozome.jgi.doe.gov). The heatmap shows the expression variance between identified 21 Phvul-SBP genes in different plant tissues (Fig. 6).

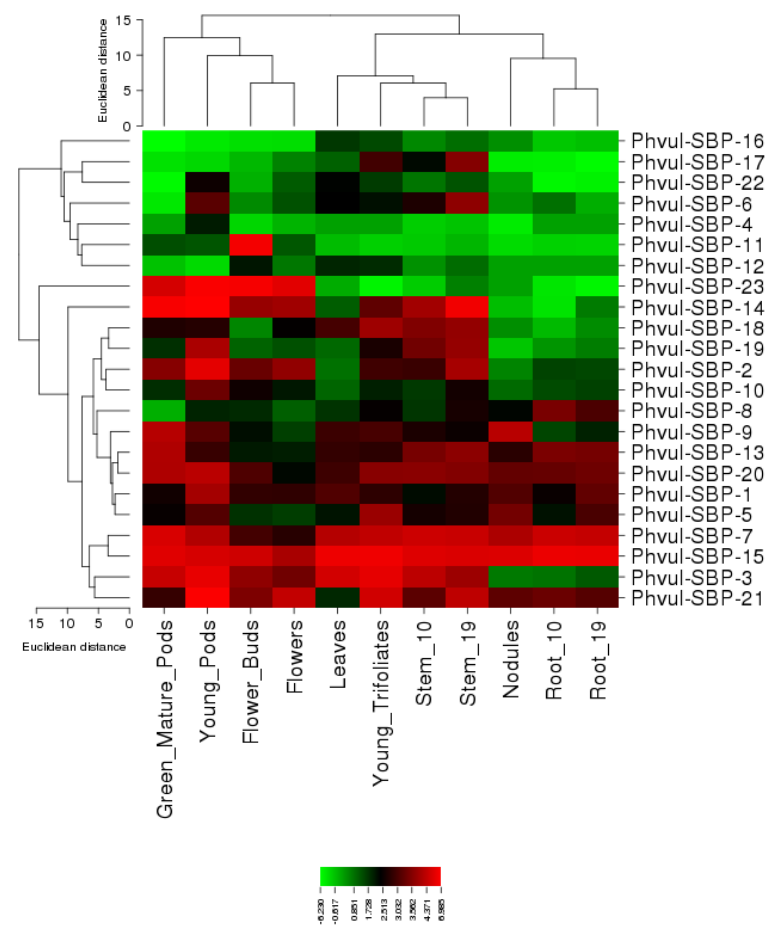

Figure 6. Hierarchical clustering of expression profiles of Phvul-SBP genes in 11 different tissues of common bean. Heat map was drawn with CIMminer online tool

As seen in the Figure 6, the Phvul-SBP-16, -17, -22, -6, -4, -11 and -12 genes revealed relatively low expression levels in almost all tissues including green mature pods, young pods, flower buds, flowers, leaves, young trifoliates, stem_10, stem_19, nodules, root_10 and root_19. The rest of the Phvul-SBP genes exhibited relatively high mRNA levels in most of the developmental stages of common bean. Phvul-SBP-14, -15 and -23 were the genes which exhibited the highest expression levels at least in one of the 
certain tissue types among all genes. Additionally, some of the genes were only up- or down-regulated in certain tissue types. For instance, Phvul-SBP-1, -3, -5, -7, -8, -9, -13, $-15,-20$ and -21 were highly expressed in root tissues. Phvul-SBP-1, -2, -3, -5, -7, - - , -9, $-10,-13,-14,-15,-18,-19,-20$ and -21 were highly expressed in stem tissues. PhvulSBP-1, - 2, -3, -5, -7, -8, -9, -10,-13,-14, -15,-18,-19,-20,-21 and -23 were highly expressed in floral organs. The other Pvul-SBP genes were low expressed in these tissues of $P$. vulgaris. Similar results were obtained in grapevine genome (Wang et al., 2010). $S B P$ genes in this genome were up or down regulated in various tissues such as root, leaf, flower and fruit. $S B P$ genes in P. vulgaris were suggested to express in different tissues.

\section{Conclusion}

In this study, genome sequences, identification, conserved motifs of 23 Pvul-SBP genes were identified in $P$. vulgaris genome. The Pvul-SBPs were divided into twelve classes and 23 Pvul-SBP genes were distributed across 10 chromosomes with different densities. Also, expression levels of 23 Pvul-SBP genes in different plant tissues such as roots, leaf and floral organs were determined using in silico analysis. To the best of our knowledge, this is the first report of a genome wide analysis of the common bean SBP gene family. This study would provide beneficial information for understanding the classification and functions of the SBP genes in common bean.

\section{REFERENCES}

[1] Ali, M. A., Alia, K. B., Atif, R. M., Rasul, I., Nadeem, H. U., Shahid, A., Azeem, F. (2017): Genome-wide identification and comparative analysis of squamosa-promoter binding proteins (Sbp) transcription factor family in Gossypium raimondii and Arabidopsis thaliana. - Pakistan Journal of Botany 49: 1113-1126.

[2] Arumuganthan, K., Earle, E. (1991): Nuclear DNA content of some important plant species. - Plant Molecular Biology Reports 9: 208-218.

[3] Bailey, T. L., Boden, M., Buske, F. A., Frith, M., Grant, C. E., Clementi, L., Ren, J., Li, W. W., Noble, W. S. (2009): MEME SUITE: tools for motif discovery and searching. Nucleic Acids Research 37(suppl_2): W202-W208.

[4] Bailey, T. L., Williams, N., Misleh, C., Li, W. W. (2006): MEME: discovering and analyzing DNA and protein sequence motifs. - Nucleic Acids Research 34: W369-W373.

[5] Bowers, J. E., Chapman, B. A., Rong, J., Paterson, A. H. (2003): Unravelling angiosperm genome evolution by phylogenetic analysis of chromosomal duplication events - Nature 422(6930): 433-8.

[6] Broughton, W. J., Hernandez, G., Blair, M., Beebe, S., Gepts, P., Vanderleyden, J. (2003): Beans (Phaseolus spp.) - model food legumes. - Plant and Soil 252(1): 55-128.

[7] Buyuk, I., Inal, B., Ilhan, E., Tanriseven, M., Aras, S., Erayman, M. (2016): Genomewide identification of salinity responsive HSP70s in common bean. - Molecular Biology Reports 43: 1251-1266.

[8] Cannon, S. B., Mitra, A., Baumgarten, A., Young, N. D., May, G. (2004): The roles of segmental and tandem gene duplication in the evolution of large gene families in Arabidopsis thaliana. - BMC Plant Boilogy 4(1): 10.

[9] Chuck, G., Whipple, C., Jackson, D., Hake, S. (2010): The maize SBP-box transcription factor encoded by tasselsheath4 regulates bract development and the establishment of meristem boundaries. - Development 137: 1585-1585. 
[10] Flagel, L. E., Wendel, J. F. (2009): Gene duplication and evolutionary novelty in plants. New Phytologist 183: 557-564.

[11] Gandikota, M., Birkenbihl, R. P., Hohmann, S., Cardon, G. H., Saedler, H., Huijser, P. (2007): The miRNA156/157 recognition element in the 3' UTR of the Arabidopsis SBP box gene SPL3 prevents early flowering by translational inhibition in seedlings. - Plant Journal 49: 683-693.

[12] Gu, Z. L., Steinmetz, L. M., Gu, X., Li, W. H. (2003): Role of duplicate genes in genetic robustness against null mutations. - Nature 421: 63-6.

[13] Guo, A., Zhu, Q., Chen, X., Luo, J. (2007): GSDS: a gene structure display server. Yi chuan. - Hereditas 29: 1023-1026.

[14] Hou, H. M., Li, J., Gao, M., Singer, S. D., Wang, H., Mao, L. Y., Fei, Z. J., Wang, X. P. (2013): Genomic organization, phylogenetic comparison and differential expression of the SBP-box family genes in grape. - Plos One 8.

[15] Ilhan, E., Buyuk, I., Inal, B. (2018): Transcriptome - Scale characterization of salt responsive bean TCP transcription factors. - Gene 642: 64-73.

[16] Inal, B., Buyuk, I., Ilhan, E. and Aras, S. (2017): Genome-wide analysis of Phaseolus vulgaris $\mathrm{C} 2 \mathrm{C} 2-\mathrm{YABBY}$ transcription factors under salt stress conditions. - 3Biotech 7(5): 302 .

[17] Jung, J. H., Ju, Y., Seo, P. J., Lee, J. H., Park, C. M. (2012): The SOC1-SPL module integrates photoperiod and gibberellic acid signals to control flowering time in Arabidopsis. - Plant Journal 69: 577-588.

[18] Juretic, N., Hoen, D. R., Huynh, M. L., Harrison, P. M., Bureau, T. E. (2005): The evolutionary fate of MULE-mediated duplications of host gene fragments in rice. Genome Research 15: 1292-1297.

[19] Kavas, M., Kızıldoğan, A. K., Abanoz, B. (2017): Comparative genome-wide phylogenetic and expression analysis of SBP genes from potato (Solanum tuberosum). Computational Biology and Chemistry 67: 131-140.

[20] Kropat, J., Tottey, S., Birkenbihl, R. P., Depege, N., Huijser, P., Merchant, S. (2005): A regulator of nutritional copper signaling in Chlamydomonas is an SBP domain protein that recognizes the GTAC core of copper response element. - Proceedings of the National Academy of Sciences of the United States of America 102: 18730-18735.

[21] Kryazhimskiy, S., Plotkin, J. B. (2008): The population genetics of dN/dS. - Plos Genetics 4.

[22] Lannenpaa, M., Janonen, I., Holtta-Vuori, M., Gardemeister, M., Porali, I. and Sopanen, T. (2004): A new SBP-box gene BpSPL1 in silver birch (Betula pendula). - Physiologia Plantarum 120: 491-500.

[23] Lee, T. H., Tang, H. B., Wang, X. Y., Paterson, A. H. (2013): PGDD: a database of gene and genome duplication in plants. - Nucleic Acids Research 41: D1152-D1158.

[24] Li, J., Hou, H. M., Li, X. Q., Xiang, J., Yin, X. J., Gao, H., Zheng, Y., Bassett, C. L., Wang, X. P. (2013): Genome-wide identification and analysis of the SBP-box family genes in apple (Malus $\times$ domestica Borkh.). - Plant Physiology and Biochemistry 70: 100-114.

[25] Li, M., Zhao, S. Z., Zhao, C. Z., Zhang, Y., Xia, H., Lopez-Baltazar, J., Wan, S. B., Wang, X. J. (2016): Cloning and characterization of SPL-family genes in the peanut (Arachis hypogaea L.). - Genetics and Molecular Research 15.

[26] Lu, S. F., Yang, C. M., Chiang, V. L. (2011): Conservation and diversity of MicroRNAassociated copper-regulatory networks in Populus trichocarpa. - Journal of Integrative Plant Biology 53: 879-891.

[27] Mao, H. D., Yu, L. J., Li, Z. J., Yan, Y., Han, R., Liu, H., Ma, M. (2016): Genome-wide analysis of the SPL family transcription factors and their responses to abiotic stresses in maize. - Plant Gene 6: 1-12. 
[28] Pan, F., Wang, Y., Liu, H. L., Wu, M., Chu, W. Y., Chen, D. M., Xiang, Y. (2017): Genome-wide identification and expression analysis of SBP-like transcription factor genes in Moso Bamboo (Phyllostachys edulis). - BMC Genomics 18: 486.

[29] Preston, J. C., Jorgensen, S. A., Orozco, R., Hileman, L. C. (2016): Paralogous SQUAMOSA PROMOTER BINDING PROTEIN-LIKE (SPL) genes differentially regulate leaf initiation and reproductive phase change in petunia. - Planta 243: 429-440.

[30] Quevillon, E., Silventoinen, V., Pillai, S., Harte, N., Mulder, N., Apweiler, R., Lopez, R. (2005): InterProScan: protein domains identifier. - Nucleic Acids Research 33: W116W120.

[31] Rhoades, M. W., Reinhart, B. J., Lim, L. P., Burge, C. B., Bartel, B., Bartel, D. P. (2002): Prediction of plant microRNA targets. - Cell 110: 513-520.

[32] Salinas, M., Xing, S. P., Hohmann, S., Berndtgen, R., Huijser, P. (2012): Genomic organization, phylogenetic comparison and differential expression of the SBP-box family of transcription factors in tomato. - Planta 235: 1171-1184.

[33] Schmutz, J., McClean, P. E., Mamidi, S., Wu, G. A., Cannon, S. B., Grimwood, J., Jenkins, J., Shu, S. Q., Song, Q. J., Chavarro, C., Torres-Torres, M., Geffroy, V., Moghaddam, S. M., Gao, D. Y., Abernathy, B., Barry, K., Blair, M., Brick, M. A., Chovatia, M., Gepts, P., Goodstein, D. M., Gonzales, M., Hellsten, U., Hyten, D. L., Jia, G. F., Kelly, J. D., Kudrna, D., Lee, R., Richard, M. M. S., Miklas, P. N., Osorno, J. M., Rodrigues, J., Thareau, V., Urrea, C. A., Wang, M., Yu, Y., Zhang, M., Wing, R. A., Cregan, P. B., Rokhsar, D. S., Jackson, S. A. (2014): A reference genome for common bean and genome-wide analysis of dual domestications. - Nature Genetics 46: 707-713.

[34] Schwarz, S., Grande, A. V., Bujdoso, N., Saedler, H., Huijser, P. (2008): The microRNA regulated SBP-box genes SPL9 and SPL15 control shoot maturation in Arabidopsis. Plant Molecular Biology 67: 183-195.

[35] Shalom, L., Shlizerman, L., Zur, N., Doron-Faigenboim, A., Blumwald, E., Sadka, A. (2015): Molecular characterization of SQUAMOSA PROMOTER BINDING PROTEINLIKE (SPL) gene family from Citrus and the effect of fruit load on their expression. Frontiers in Plant Science 6: 389.

[36] Song, A., Gao, T., Wu, D., Xin, J., Chen, S., Guan, Z., Wang, H., Jin, L., Chen, F. (2016): Transcriptome-wide identification and expression analysis of chrysanthemum SBP-like transcription factors. - Plant Physiology and Biochemistry 102: 10-16.

[37] Tamura, K., Stecher, G., Peterson, D., Filipski, A., Kumar, S. (2013): MEGA6: Molecular Evolutionary Genetics Analysis Version 6.0. - Molecular Biology and Evolution 30: 2725-2729.

[38] Unte, U. S., Sorensen, A. M., Pesaresi, P., Gandikota, M., Leister, D., Saedler, H., Huijser, P. (2003): SPL8, an SBP-Box gene that affects pollen sac development in Arabidopsis. - Plant Cell 15: 1009-1019.

[39] Voorrips, R. E. (2002): MapChart: Software for the graphical presentation of linkage maps and QTLs. - Journal of Heredity 93: 77-78.

[40] Wang, B. N., Geng, S. F., Wang, D., Feng, N., Zhang, D. D., Wu, L., Hao, C. Y., Zhang, X. Y., Li, A. L., Mao, L. (2015): Characterization of SQUAMOSA promoter binding protein-like genes in wheat. - Journal of Plant Biology 58: 220-229.

[41] Wang, Y., Hu, Z., Yang, Y., Chen, X., Chen, G. (2010): Genome-wide identification, phylogeny, and expression analysis of the SBP-box gene family in grapevine. - Russian Journal of Plant Physiology 57: 273-282.

[42] Xie, K. B., Wu, C. Q., Xiong, L. Z. (2006): Genomic organization, differential expression, and interaction of SQUAMOSA promoter-binding-like transcription factors and microRNA156 in rice. - Plant Physiology 142: 280-293.

[43] Yang, Z. H. (2007): PAML 4: Phylogenetic analysis by maximum likelihood. Molecular Biology and Evolution 24: 1586-1591. 
[44] Yang, Z. H., Swanson, W. J. (2002): Codon-substitution models to detect adaptive evolution that account for heterogeneous selective pressures among site classes. Molecular Biology and Evolution 19: 49-57.

[45] Zhang, H. X., Jin, J. H., He, Y. M., Lu, B. Y., Li, D. W., Chai, W. G., Khan, A., Gong, Z. H. (2016): Genome-wide identification and analysis of the SBP-box family genes under Phytophthora capsici stress in pepper (Capsicum annuum L.). - Frontiers in Plant Science 7: 504.

[46] Zhang, L. S., Wu, B., Zhao, D. G., Li, C. L., Shao, F. J., Lu, S. F. (2014): Genome-wide analysis and molecular dissection of the SPL gene family in Salvia miltiorrhiza. - Journal of Integrative Plant Biology 56: 38-50.

[47] Zhang, X. H., Dou, L. L., Pang, C. Y., Song, M. Z., Wei, H. L., Fan, S. L., Wang, C. S., Yu, S. X. (2015): Genomic organization, differential expression, and functional analysis of the SPL gene family in Gossypium hirsutum. - Molecular Genetics and Genomics 290: $115-126$.

[48] Zhang, Y., Schwarz, S., Saedler, H., Huijser, P. (2007): SPL8, a local regulator in a subset of gibberellin-mediated developmental processes in Arabidopsis. - Plant Molecular Biology 63: 429-439.

[49] Zheng, Y., Jiao, C., Sun, H. H., Rosli, H. G., Pombo, M. A., Zhang, P. F., Banf, M., Dai, X. B., Martin, G. B., Giovannoni, J. J., Zhao, P. X., Rhee, S. Y., Fei, Z. J. (2016): iTAK: A program for genome-wide prediction and classification of plant transcription factors, transcriptional regulators, and protein kinases. - Molecular Plant 9: 1667-1670. 